\title{
String Breaking in Quenched QCD
}

\author{
Chris Stewart and Roman Koniuk, \\ Physics and Astronomy, York University, Toronto, Ontario, M3J 1P3 Canada.
}

We present preliminary quenched results on a new operator for the investigation of string-breaking within $\mathrm{SU}(2)$-colour QCD. The ground-state of a spatially-separated static-light meson-antimeson pair is a combination of a state with two distinct mesons, expected to dominate for large separations, and a state where the lightquarks have annihilated, which contributes for short distances. The crossover between these two regimes provides a measure of the string-breaking scale length.

\section{INTRODUCTION}

A first-principles demonstration of stringbreaking has eluded lattice QCD practitioners for many years. Traditionally, researchers have performed Wilson loop simulations in full QCD, searching for a plateau in the static quark potential that would signal the onset of string-breaking. The lack of evidence has led some to suggest that the Wilson loop operator may have too small an overlap with the broken two-meson state [1.2], and to recommend a search for better operators.

In this paper, we suggest such an operator, consisting of a product of spatially-separated staticlight meson operators, that provides superior overlap with the broken-string state. We also present preliminary results that indicate this operator does induce string-breaking, even within the quenched approximation.

\section{THE OPERATOR}

The standard operator used in string-breaking investigations is the Wilson loop, the propagator for a spatially-separated static quark-antiquark pair. Obviously this operator has strong overlap with the unbroken state of two static quarks joined by a gluon flux tube - sadly, it has proven to have insufficient overlap with the broken state of two distinct static-light mesons.

Consider then, a composite operator consisting of a static-light meson-antimeson pair, separated by a distance $\vec{R}$,

$\mathcal{O}_{M \bar{M}}(\vec{R})=\bar{\psi}_{l}(0) \gamma_{5} \psi_{S}(0) \bar{\psi}_{S}(\vec{R}) \gamma_{5} \psi_{l}(\vec{R})$.
The meson-pair propagator is

$$
G_{M \bar{M}}(t, \vec{R})=\mathcal{G}_{D}+\mathcal{G}_{E},
$$

where

$$
\begin{aligned}
\mathcal{G}_{D}(t, \vec{R})= & \operatorname{Tr}\left[G_{h}(0, t ; 0,0) G_{l}^{\dagger}(0, t ; 0,0)\right] \\
\times & \operatorname{Tr}\left[G_{l}(\vec{R}, t ; \vec{R}, 0) G_{h}^{\dagger}(\vec{R}, t ; \vec{R}, 0)\right], \\
\mathcal{G}_{E}(t, \vec{R})= & -\operatorname{Tr}\left[G_{h}(0, t ; 0,0) G_{l}^{\dagger}(\vec{R}, t ; 0,0)\right. \\
& \left.G_{h}^{\dagger}(\vec{R}, t ; \vec{R}, 0) G_{l}(0, t ; \vec{R}, 0)\right] .
\end{aligned}
$$

Contributions to $\mathcal{G}_{D}$ and $\mathcal{G}_{E}$, the 'direct' and 'exchange' terms, are depicted in Figure 1.

The large- $R$ limit of this system must be a state with two distinct mesons, and so we expect $\mathcal{G}_{D}$ will dominate for large separations. For small $R$, however, the light quarks can easily annihilate, as shown in Figure 1, leaving a static quarkantiquark pair interacting through the gluon field. The exchange term $\mathcal{G}_{E}$ should contribute strongly for small separations, giving the necessary overlap with the unbroken state before string-breaking occurs.

\section{THE SIMULATION}

We performed a preliminary simulation to test the validity of the $M \bar{M}$ operator. An ensemble of 150 quenched $\mathrm{SU}(2)$-colour gauge configurations was created using an $\mathcal{O}\left(a^{2}\right)$-improved action,

$S_{G}=-\beta \sum_{x, \mu>\nu}\left(\frac{5}{3} \frac{P_{\mu \nu}}{u_{0}^{4}}-\frac{1}{12} \frac{R_{\mu \nu}+R_{\nu \mu}}{u_{0}^{6}}\right)$.

where $P_{\mu \nu}$ is the plaquette operator, $R_{\mu \nu}$ is a $2 \times 1$ loop with the long side along the $\mu$-direction. 

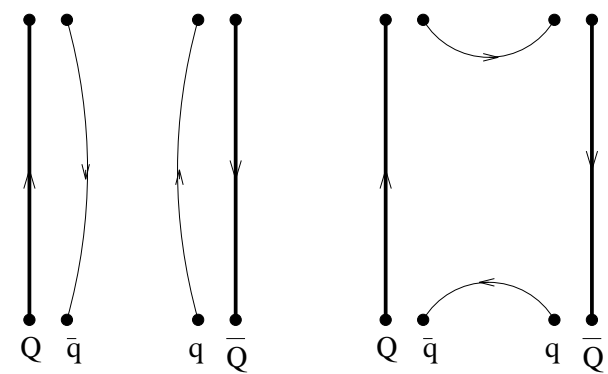

Figure 1. Contributions to direct and exchange terms - note the annihilation of light quarks in the exchange diagram.

The lattice dimensions were $\left(L_{x}, L_{y}, L_{z}, L_{t}\right)=$ $(10,8,8,12)$, with the separation between the static quarks along the $\mathrm{x}$-direction. The tadpole correction $u_{0}$ was derived from the plaquette operator,

$u_{0}=\left\langle P_{\mu \nu}\right\rangle^{1 / 4}$.

The simulation was performed at $\beta=1.07$, corresponding to a lattice spacing of roughly $0.2 \mathrm{fm}$, using the $\rho$ - and $\pi$-meson mass ratio to set the scale.

We used the tadpole-improved SheikholeslamiWohlert operator for the fermion action,

$M_{S W}=m_{0}+\sum_{\mu}\left(\gamma_{\mu} \triangle_{\mu}-\frac{1}{2} \triangle_{\mu}^{2}\right)-\frac{1}{4} \sigma \cdot F$.

Due to the computational complexity of this problem, the simulation was performed at a very high quark mass, $\kappa=0.135$, corresponding to a pion to rho-meson mass ratio of $m_{\pi} / m_{\rho} \simeq 0.75$.

\section{RESULTS}

Figure 2 shows a comparison of the direct and exchange terms in the propagator (2) for varying separation $R$. Also shown is a linear-pluscoulomb fit to the Wilson loop data from the same lattice ensemble. Note that for small R, the exchange term gives a contribution almost identical in size to the Wilson loop potential, indicating

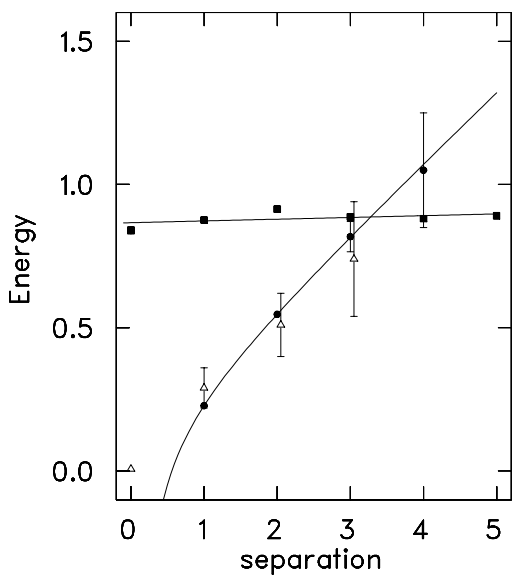

Figure 2. Direct term (squares), exchange term (triangles) and Wilson loop potential (circles and solid line). Exchange term data are offset for clarity.

that the light quarks are indeed annihilating to leave a static quark-antiquark pair.

We expect string-breaking to occur at the point where the two-meson state becomes energetically favourable - from the slope of the Wilson loop potential, this appears to be between $R=3$ and $R=4$. Unfortunately, noise overcomes the exchange term signal just at this point, and the crossover can only be inferred from the Wilson loop data.

The full propagator, shown in Figure 3, gives a much clearer picture. The system's energy increases to a plateau at the expected level of the mass of the two separate mesons. The small error bars for large values of $R$ indicate that, although noise has destroyed the exchange term's signal, the mixing into this term has vanished.

Figure 4 confirms this to be the case. The comparative sizes of $\mathcal{G}_{D}(t=0)$ and $\mathcal{G}_{E}(t=0)$ are taken as a rough measure of the mixing into each of the direct and exchange terms, and are plotted as a function of $R$. As expected, the direct and exchange terms both contribute for small $R$, and the exchange term vanishes quickly as sepa- 


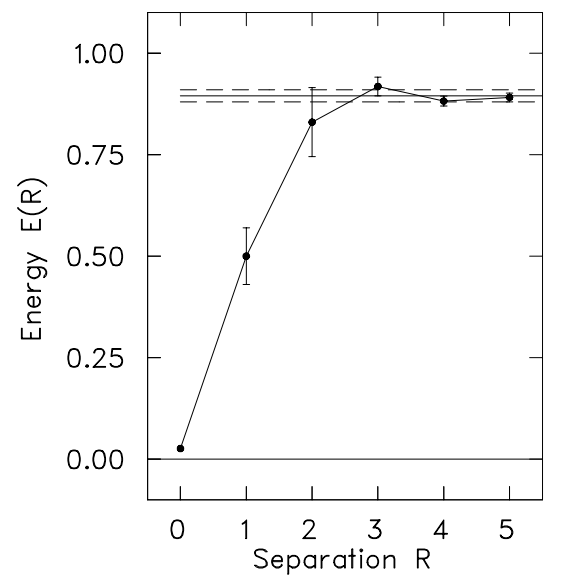

Figure 3. Potential from full propagator. Horizontal line is mass of free static-light meson.

ration increases, leaving the direct term to dominate completely for large $R$.

\section{CONCLUSIONS}

The use of new operators, or combinations of operators, to demonstrate string-breaking on the lattice appears to be an idea whose time has arrived - witness the flood of authors in these proceedings presenting results in $S U(2)$ QCD with scalar fields [3 5], and references to ongoing research in full $S U(3)$ QCD [6].

We have described an operator suitable for use in string-breaking investigations, combining the short-range and long-range physics into a single operator - that of a spatially separated staic-light meson pair. For small separations, this operator behaves like a Wilson loop, resulting in a confining potential. For larger separations, the potential reaches a plateau at the energy of two distinct static-light mesons, the fingerprint of the elusive broken string. This occurs even in a quenched simulation, since the operator must energetically favour the meson-pair state for large $R$, and so forces the gluon string to break. The light quarks are providing, within the quenched approxima-

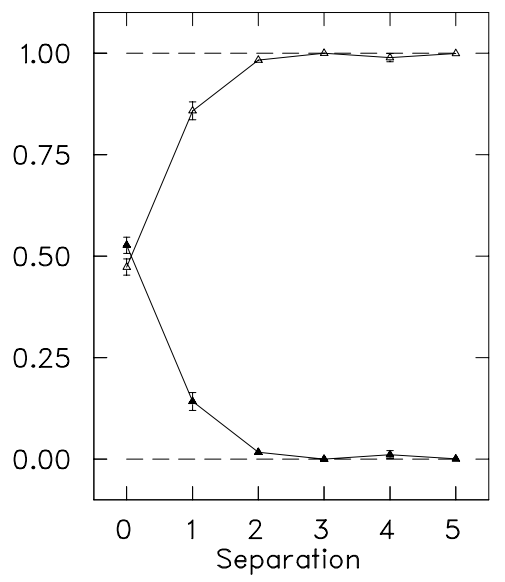

Figure 4. Overlap with direct term (open) and exchange term (closed).

tion, some sea-quark effects.

While we are acutely aware of the limitations of the results described here, we are confident that this operator will allow accurate determination of the string-breaking distance when used in more ambitious simulations.

We thank Howard Trottier and Norm Shakespeare for helpful discussions and suggestions. This work was supported in part by the National Sciences and Engineering Research Council of Canada.

\section{REFERENCES}

1. P. de Forcrand, these proceeings.

2. S. Güsken, Nuc. Phys. B (Proc. Suppl.) 63 (1998) 16.

3. H. Trottier, these proceedings.

4. O. Philipsen and H. Wittig, these proceedings and hep-lat/9807020.

5. F. Knechtli and R. Sommer, these proceedings and hep-lat/9807022.

6. P. Pennanen, these proceedings. 